MedTech Pilot Program with the goal of translating discoveries into novel health technologies that address important unmet health needs. The MedTech Pilot Program is an innovative funding mechanism that seeks to (1) stimulate clinical translational research, (2) help promising projects bridge the gap between the bench and the patients' bedside, and (3) encourage collaborative, transdisciplinary work. Specifically, the Pilot Program offers up to $\$ 50,000$ to support projects involving medical devices and mobile technologies used for (1) therapeutic applications and (2) device-based patient-specific (or POC) diagnostic applications. This analysis of the MedTech Pilot Program will: 1) describe the Program's structure and process; 2) highlight the intensive, hands-on mentorship and practical guidance awardees receive that enables them to more efficiently and effectively advance their projects toward patient care; and 3) characterize the progress of the 36 funded projects. METHODS/STUDY POPULATION: Key elements of the Pilot Program's infrastructure and mentoring processes as they relate to project outcomes were identified. Additionally, outcomes data were collected from two sources: (1) annual survey of Pilot Awardees and (2) publicly available information relevant to the pilot projects. RESULTS/ANTICIPATED RESULTS: The Pilot Program's framework and infrastructure has supported a diverse group of transdisciplinary projects. These projects were evaluated using both traditional and non-traditional metrics (e.g., patents, startups, publications). The initial investment of $\$ 1.5$ million to fund 36 projects has led to over $\$ 88$ million dollars in additional funding. Additionally, taking full advantage of the expertise in Silicon Valley, strong mentorship has helped advance projects along the clinical and translational path. DISCUSSION/ SIGNIFICANCE OF IMPACT: The Pilot Program has benefited Stanford innovators and researchers by providing seed funding to help promising projects bridge the gap between the bench and the bedside. The intensive, hands-on mentorship, early pilot funding, and practical guidance pilot awardees receive effectively help translate their technologies into patient care.

3030

\section{Symptom profile of chronic rhinosinusitis versus obstructive sleep apnea in a tertiary rhinology clinic} Keven Seung Yong $\mathrm{Ji}^{1}$, Thomas J. Risoli, Maragatha Kuchibhatla, Lyndon Chan, Ralph Abi Hachem and David Jang

${ }^{1}$ Duke University

OBJECTIVES/SPECIFIC AIMS: Patients with undiagnosed obstructive sleep apnea (OSA) will often present to an otolaryngologist with symptoms of chronic rhinosinusitis (CRS). Differentiating CRS from OSA may help obviate unnecessary and costly work-up for CRS. This study analyzes symptom profiles of such patients to help identify which require polysomnography. METHODS/STUDY POPULATION: This is a three-year retrospective analysis of adult patients seen in an academic practice with a rhinologic chief complaint. The 22-Item Sinonasal Outcomes Test (SNOT-22) survey, which is a validated patient-reported outcome measure widely adopted for CRS featuring a symptom scale of 1 (least severe) to 5 (most severe), was completed by patients with untreated OSA confirmed on polysomnography without CRS (OSA group) and a control group of CRS patients (CRS group). Results were compared using Chi-square test (categorical) and Wilcoxon rank-sum test (continuous) with Bonferroni correction, and multiple logistic regression. RESULTS/ANTICIPATED RESULTS: 43 patients were included in the OSA group [mean apnea-hypopnea index: 27.9 (SD: 21.2)] and 124 patients were included in the CRS group.
The CRS group demonstrated significantly higher scores in nasal ( $\mathrm{p}<0.001)$, extra-nasal $(\mathrm{p}<0.001)$ and ear/facial symptom domains $(\mathrm{p}=0.001)$ while the OSA group reported higher psychological $(p=0.028)$ and sleep symptom domain scores $(p=0.052)$. As for the cardinal symptoms of CRS, nasal discharge and loss of smell were significantly higher in the CRS group (both $p<0.001$ ), whereas facial pain $(p=0.117)$ and nasal obstruction $(p=0.198)$ were not significantly different between the two groups. After adjustment, for every 1-point increase in a patient's score for ear pain, thick nasal discharge and loss of smell or taste, their odds of having CRS increased by a factor of 3.18 [(95\% CI 1.61-6.29), $\mathrm{p}=0.001$ ], 1.60 [(95\% CI 1.22 2.10 ], $\mathrm{p}=0.001$ ] and 1.36 [(95\% CI 1.04-1.78), $\mathrm{p}=0.025$ ], respectively, compared to having OSA. OSA patients were more likely to choose a sleep-related symptom as a "most important complaint" (MIC) $(\mathrm{p}<0.001)$. Facial pain and nasal obstruction were the most common MIC in the rhinologic domain for OSA patients, whereas thick nasal discharge and post-nasal discharge were the most common MIC for CRS patients. DISCUSSION/SIGNIFICANCE OF IMPACT: For patients presenting with rhinologic symptoms, the SNOT-22 can help identify those with undiagnosed OSA. OSA should be suspected in patients with sleep and psychological dysfunction as their primary complaints without the significant nasal drainage and anosmia that characterizes CRS.

3541

The association of corticosteroid use with inpatient mortality in acute exacerbation of idiopathic pulmonary fibrosis

Erica Farrand ${ }^{1}$, Eric Vittinghoff, Brett Ley and Harold Collard ${ }^{1}$ University Of California, San Francisco

OBJECTIVES/SPECIFIC AIMS: Objective: To assess the impact of corticosteroid therapy on in-hospital mortality in IPF patients admitted with acute respiratory failure. METHODS/STUDY POPULATION: Methods: Patients with IPF were retrospectively identified in the University of California San Francisco medical center's electronic health records from January 1, 2010 to June 1, 2018. Cases with IPF were defined as age 50 years or older, having at least two codes one month apart for idiopathic fibrosing alveolitis or postinflammatory fibrosis (ICD-9 516.3, 516.31 or 515.0 or ICD-10 codes J84.9, J84.10, J84.111 or J84.112), and a subsequent hospitalization for acute respiratory failure or acute respiratory symptoms. The prevalence of pre-selected co-morbidities, clinical events (ICU admission, mechanical ventilation, lung transplantation) and clinical outcomes were assessed. A propensity score model for corticosteroid use was constructed using a multivariable logistic regression with inclusion of corticosteroid-associated demographic and baseline variables (univariate $\mathrm{p}$-value $<0.25$ ). A marginal structural model (MSM) was used to address time-dependent confounding and mediating effects of ICU admission and mechanical ventilation by applying inverse probability weighting for receipt of corticosteroid treatment. Secondary outcome analysis was performed on patients who survived hospital admission. RESULTS/ANTICIPATED RESULTS: Results: A total of 132 patients with IPF and an acute respiratory admission were identified. 48 patients (36\%) received corticosteroids during their admission. Applying inverse weighting to time-dependent co-variates (ICU admission and invasive mechanical ventilation) in a MSM, corticosteroid therapy was not associated with risk of in-hospital mortality (odds ratio 1.82; 95\% CI, 0.47-6.99; $\mathrm{p}=0.39$ ). After adjusting for corticosteroid therapy using a propensity score, corticosteroid therapy remained unassociated 